\title{
Closed graphs are proper interval graphs
}

\author{
Marilena Crupi and Giancarlo Rinaldo
}

\begin{abstract}
Let $G$ be a connected simple graph. We prove that $G$ is a closed graph if and only if $G$ is a proper interval graph. As a consequence we obtain that there exist linear-time algorithms for closed graph recognition.
\end{abstract}

\section{Introduction}

In this note a graph $G$ means a connected simple graph without isolated vertices, that is, $G$ is connected without loops and multiple edges. Let $V(G)=$ $[n]=\{1, \ldots, n\}$ be the set of vertices and $E(G)$ the edge set of $G$.

Let $S=K\left[x_{1}, \cdots, x_{n}, y_{1}, \cdots, y_{n}\right]$ be the polynomial ring in $2 n$ variables with coefficients in a field $K$. For $i<j$, set $f_{i j}=x_{i} y_{j}-x_{j} y_{i}$. The ideal $J_{G}$ of $S$ generated by the binomials $f_{i j}=x_{i} y_{j}-x_{j} y_{i}$ such that $i<j$ and $\{i, j\}$ is an edge of $G$, is called the binomial edge ideal of $G$. Such class of ideals is a generalization of the ideal of 2 -minors of a $2 n$-matrix of indeterminates. In fact, the ideal of 2 -minors of a $2 n$-matrix may be considered as the binomial edge ideal of a complete graph on $[n]$. The relevance of this class of ideals for algebraic statistics is underlined in [14]. Indeed these ideals arise naturally in the study of conditional independence statements [5]. If $\prec$ is a monomial order on $S$, then a graph $G$ on the vertex set $[n]$ is closed with respect to the given labelling of the vertices if the generators $f_{i j}$ of $J_{G}$ form a quadratic Gröbner basis $[14,4]$.

A combinatorial description of this fact is the following. A graph $G$ is closed with respect to the given labelling of the vertices if the following condition

Key Words: Closed graphs, interval graphs, Gröbner bases.

2010 Mathematics Subject Classification: Primary 05C25. Secondary 13 C05.

Received: 18 September, 2013

Accepted: 13 November, 2013 
is satisfied: for all edges $\{i, j\}$ and $\{k, \ell\}$ with $i<j$ and $k<\ell$, one has $\{j, \ell\} \in E(G)$ if $i=k$, and $\{i, k\} \in E(G)$ if $j=\ell$.

In particular, $G$ is closed if there exists a labelling for which it is closed.

In the last years different authors $[14,16,4,19]$ concentrated their attention on the class of closed graphs. The most recent characterization of this class of graphs is given in [3], where it is proved that a connected graph has a closed labeling if and only if it is chordal, $K_{1,3}$-free, and has a property called narrow, which holds when every vertex is distance at most one from all longest shortest paths of the graph.

In [4] we have conjectured that by a suitable ordering on the vertices it is possible to test the closedness of a graph in linear time. In this note we are able to prove the conjecture.

In the research of a linear-time algorithm for closed graph recognition we have observed that the class of closed graphs and the class of proper interval graphs are the same.

Proper interval graphs are the intersection graphs of intervals of the real line where no interval properly contains another and have been extensively studied since their inception $[10,12]$. There are several representations and many characterizations of them $[8,13,18]$ and some of them through vertex orderings. Such class of graphs has many applications, such as physical mapping of DNA and genome reconstruction $[25,9]$.

During the last decade, many linear-time recognition algorithms for proper interval graphs have been developed $[2,20,17,22]$ and most of them are based on special breadth-first search (BFS) strategies.

The first linear-time algorithm for interval graph recognition appeared in 1976 [1]. This algorithm uses a lexicographic breadth first search (lexBFS) to find in linear time the maximal cliques of the graphs and then employs special structure called $P Q$-trees to find an ordering of the maximal cliques that characterizes interval graphs. A lexBFS is a breadth first search procedure with the additional rule that vertices with earlier visited neighbors are preferred and its vantage is that it can be performed in $O(|V(G)|+|E(G)|)$ time [24].

The paper is organized as follows. Section 1 contains some preliminaries and notions that will be used in the paper. In Section 2, we prove our conjecture (Theorem 2.4): Let $G$ be a graph. $G$ is a closed graph if and only if $G$ is a proper interval graph.

As a consequence we are able to state that by an ordering on the vertices obtained by a lexBFS research it is possible to test the closedness of a graph in linear-time. 


\section{Preliminaries}

In this Section we recall some concepts and a notation on graphs and simplicial complexes that we will use in the article.

Let $G$ be a graph with vertex set $V(G)$ and edge set $E(G)$.

When we fix a given labelling on the vertices we say that $G$ is a graph on $[n]$.

Let $G$ be a graph with vertex set $[n]$. A subset $C$ of $[n]$ is called a clique of $G$ is for all $i$ and $j$ belonging to $C$ with $i \neq j$ one has $\{i, j\} \in E(G)$.

Two graphs $G$ and $H$ are isomorphic if there exists a bijection between the vertex sets of $G$ and $H$, namely $\phi: V(G) \rightarrow V(H)$, such that $\{u, v\} \in E(G)$ if and only if $\{\phi(u), \phi(v)\} \in E(H)$.

Set $V=\left\{x_{1}, \ldots, x_{n}\right\}$. A simplicial complex $\Delta$ on the vertex set $V$ is a collection of subsets of $V$ such that

(i) $\left\{x_{i}\right\} \in \Delta$ for all $x_{i} \in V$ and

(ii) $F \in \Delta$ and $G \subseteq F$ imply $G \in \Delta$.

An element $F \in \Delta$ is called a face of $\Delta$. A maximal face of $\Delta$ with respect to inclusion is called a facet of $\Delta$. If $\Delta$ is a simplicial complex with facets $F_{1}, \ldots, F_{q}$, we write $\Delta=\left\langle F_{1}, \ldots, F_{q}\right\rangle$.

Definition 1.1. The clique complex $\Delta(G)$ of $G$ is the simplicial complex whose faces are the cliques of $G$.

The clique complex plays an important role in the study of the class of closed graphs $[14,4]$.

Definition 1.2. A graph $G$ is closed with respect to the given labelling if the following condition is satisfied:

for all edges $\{i, j\}$ and $\{k, \ell\}$ with $i<j$ and $k<\ell$ one has $\{j, \ell\} \in E(G)$ if $i=k$, and $\{i, k\} \in E(G)$ if $j=\ell$.

In particular, $G$ is closed if there exists a labelling for which it is closed.

Theorem 1.3. Let $G$ be a graph. The following conditions are equivalent:

(1) there exists a labelling $[n]$ of $G$ such that $G$ is closed on $[n]$;

(2) $J_{G}$ has a quadratic Gröbner basis with respect to some term order $\prec$ on $S$;

(3) there exists a labelling of $G$ such that all facets of $\Delta(G)$ are intervals $[a, b] \subseteq[n]$.

Proof. (1) $\Leftrightarrow(2)$ : see [4], Theorem 3.4.

(1) $\Leftrightarrow(3)$ : see [16], Theorem 2.2. 


\section{The result}

In this Section we prove that closed graphs are proper interval graphs and viceversa.

Definition 2.1. A graph $G$ is an interval graph if to each vertex $v \in V(G)$ a closed interval $I_{v}=\left[\ell_{v}, r_{v}\right]$ of the real line can be associated, such that two distinct vertices $u, v \in V(G)$ are adjacent if and only if $I_{u} \cap I_{v} \neq \emptyset$.

The family $\left\{I_{v}\right\}_{v \in V(G)}$ is an interval representation of $G$.

Definition 2.2. A graph $G$ is a proper interval graph if there is an interval representation of $G$ in which no interval properly contains another.

If $G$ is a graph, a vertex ordering $\sigma$ for $G$ is a permutation of $V(G)$. We write $u \prec_{\sigma} v$ if $u$ appears before $v$ in $\sigma$.

Ordering $\sigma$ is called a proper interval ordering if for every triple $u, v, w$ of vertices of $G$ where $u \prec_{\sigma} v \prec_{\sigma} w$ and $\{u, w\} \in E(G)$, one has $\{u, v\},\{v, w\} \in$ $E(G)$. This condition is called the umbrella property [13].

The vertex orderings allow to state many characterizations of proper interval graphs. We quote the next result from [18, Theorem 2.1].

Theorem 2.3. A graph $G$ is a proper interval graph if and only if $G$ has a proper interval ordering.

Now we are in position to state and prove the result of the paper.

Theorem 2.4. Let $G$ be a graph. The following conditions are equivalent:

(1) $G$ is a closed graph;

(2) $G$ is a proper interval graph.

Proof. Since a graph $G$ is closed if and only if each connected component is closed we may assume that the graph $G$ is connected.

$(1) \Rightarrow(2)$. Let $G$ be a closed graph.

Claim 1. There exists a proper interval graph $H$ such that $G$ is isomorphic to $H$.

Since $G$ is closed then there exists a labelling $[n]$ of $G$ such that all facets of the clique complex $\Delta(G)$ are intervals $[a, b] \subseteq[n]$ (Theorem 1.3), that is

$$
\Delta(G)=\left\langle\left[a_{1}, b_{1}\right],\left[a_{2}, b_{2}\right], \ldots,\left[a_{r}, b_{r}\right]\right\rangle,
$$

with $1=a_{1}<a_{2}<\ldots<a_{r}<n, 1<b_{1}<b_{2}<\ldots<b_{r}=n$ with $a_{i}<b_{i}$ and $a_{i+1} \leq b_{i}$, for $i \in[r]$. 
Set $\varepsilon=\frac{1}{n}$. Define the following closed intervals of the real line:

$$
I_{k}=[k, b(k)+k \varepsilon],
$$

where

$$
b(k)=\max \left\{b_{i}: k \in\left[a_{i}, b_{i}\right]\right\}, \quad \text { for } k=1, \ldots, n .
$$

Let $H$ be the interval graph on the set $V(H)=\left\{I_{1}, \ldots, I_{n}\right\}$ and let

$$
\varphi: V(G)=[n] \rightarrow V(H)
$$

be the map defined as follows:

$$
\varphi(k)=I_{k} .
$$

$\varphi$ is an isomorphism of graphs.

In fact, let $\{k, \ell\} \in E(G)$ with $k<\ell$. We will show that $\{\varphi(k), \varphi(\ell)\}=$ $\left\{I_{k}, I_{\ell}\right\} \in E(H)$, that is, $I_{k} \cap I_{\ell} \neq \emptyset$.

It is

$$
I_{k}=[k, b(k)+k \varepsilon], \quad I_{\ell}=[\ell, b(\ell)+\ell \varepsilon] .
$$

Suppose $I_{k} \cap I_{\ell}=\emptyset$. Then $b(k)+k \varepsilon<\ell$ and consequently $b(k)<\ell$. It follows that does not exist a clique containing the edge $\{k, \ell\}$. A contradiction.

Now, suppose that $\left\{I_{k}, I_{\ell}\right\} \in E(H)$, with $k<\ell$. We will prove that $\{k, \ell\} \in E(G)$.

Since $I_{k} \cap I_{\ell} \neq \emptyset$, then $b(k)+k \varepsilon \geq \ell$. By the meaning of $\varepsilon$ and by the assumption $k<\ell$, it follows that $k \varepsilon<1$ and so $b(k) \geq \ell$. Hence from (2.1) and $(2.2),\{k, \ell\} \in E(G)$.

Since $G$ is closed and consequently a $K_{1,3}$-free graph [23], the isomorphism $\varphi$ assures that $H$ is a proper interval graph.

Hence $G$ is up to isomorphism a proper interval graph and (2) follows.

$(2) \Rightarrow(1)$. Let $G$ be a proper interval graph.

Claim 2. There exists a closed graph $H$ such that $G$ is isomorphic to $H$.

Let $\left\{I_{v}\right\}_{v \in V(G)}$ be an interval representation of $G$, with $|V(G)|=n$.

From Theorem 2.3, there exists a proper interval ordering $\sigma$ of $G$. Let $\sigma=\left(I_{1}, \ldots, I_{n}\right)$ be such vertex ordering. It is $I_{j} \prec_{\sigma} I_{k}$ if and only if $j<k$.

Let $H$ be the graph with vertex set $V(H)=[n]$ and edge set $E(H)=$ $\left\{\{i, j\}:\left\{I_{i}, I_{j}\right\} \in E(G)\right\}$.

We prove that $H$ is a closed graph on $[n]$.

Let $\{i, j\},\{k, \ell\} \in E(H)$ with $i<j$ and $k<\ell$. 
Suppose $i=k$. Since $\{i, j\},\{i, \ell\} \in E(H)$, then $\left\{I_{i}, I_{j}\right\},\left\{I_{i}, I_{\ell}\right\} \in E(G)$.

If $i<j<\ell$, then $I_{i} \prec_{\sigma} I_{j} \prec_{\sigma} I_{k}$. Hence since $\sigma$ satisfies the umbrella property and $\left\{I_{i}, I_{\ell}\right\} \in E(G)$, it follows that $\left\{I_{i}, I_{j}\right\},\left\{I_{j}, I_{\ell}\right\} \in E(G)$. Thus $\{j, \ell\} \in E(H)$. again.

Repeating the same reasoning for $i<\ell<j$, it follows that $\{j, \ell\} \in E(H)$

Similarly for $j=\ell$, one has $\{i, k\} \in E(H)$. Hence $H$ is a closed graph.

It is easy to verify that the proper interval graph $G$ is isomorphic to the closed graph $H$ by the map $\psi: V(G) \rightarrow V(H)=[n]$, that sends every closed interval $I_{j} \in V(G)$ to the integer $j \in V(H)$.

Hence $G$ is up to isomorphism a closed graph and (1) follows.

Remark 2.5. For the implication $(2) \Rightarrow(1)$, see also [19, Proposition 1.8]

\section{References}

[1] K.S. Booth, G.S. Lucker, Testing for the consecutive ones property, interval graphs, and graph planarity using PQ-tree algorithms, J. Comput. System Sci., 13 (1976), 335-379.

[2] D. G. Corneil, A simple 3-sweep LBFS algorithm for the recognition of unit interval graphs, Discrete Appl. Math., 138 (2004), 371-379.

[3] D. A. Cox, A. Erskine, On closed graphs, (2013) arXiv:1306.5149v1.

[4] M. Crupi, G. Rinaldo, Binomial edge ideals with quadratic Gröbner bases, Electron. J. Combin, 18 (2011) P\#211, 1-13.

[5] M. Drton, B. Sturmfels, S. Sullivan, Lectures on Algebraic statistics, Birhäuser, Boston, Cambridge, 2009.

[6] D. Eisenbud, B. Sturmfels, Binomial ideals, Duke Math. J., 84 (1996), $1-45$.

[7] C. M. H. Figueiredo, J. Meidanis, C. P. Mello, A linear-time algorithm for proper interval graph recognition, Inform. Process. Lett., 56 (3) (1995), $179-184$.

[8] F. Gardi, The Roberts characterization of proper and unit interval graphs, Discrete Math., 307(22) (2007), 2906-2908.

[9] P. W. Golberg, M. C. Golumbic, H. Kaplan, R. Shamir, Four strikes against physical mapping of DNA, J. Comput. Biol., 2 (1995), 139-152. 
[10] M. C. Golumbic, Algorithm Graph Theory and Perfect graphs, Academic Press, New York, N.Y., 1980.

[11] M. Habib, R. McConnel, C. Paul, L.Viennot, Lex-BFS and partition refinement, with applications to transitive orientation, interval graph recognition, and consecutive one testing, Theoret. Comput. Sci., 234 (2000), $59-84$

[12] G. Hajós, Über eine Art von Graphen, Internat. Math. Nachrichten, 11(1957) problem 65.

[13] P. Heggernes, D. Meister, C. Papadopoulos, A new representation of proper interval graphs with an application to clique-width, DIMAP Workshop on Algorithmic Graph Theory 2009, Electron. Notes Discrete Math., 32 (2009), 27-34.

[14] J. Herzog, T. Hibi, F. Hreinsdottir, T. Kahle, J. Rauh, Binomial edge ideals and conditional independence statements, Adv. in Appl. Math., 45 (2010), 317-333

[15] J. Herzog, T. Hibi, Monomial ideals, Grad. Texts in Math., Springer, 2010.

[16] J. Herzog, V. Ene, T. Hibi, Cohen-Macaulay binomial edge ideals, Nagoya Math. J., 204 (2011), 57-68.

[17] P. Hell and J. Huang, Certifying LexBFS Regognition Algorithms for Proper Interval graphs and proper Interval Bigraphs, SIAM J. Discrete Math., 18 (2005), 554-570.

[18] P. J. Looges, S. Olariu, Optimal greedy algorithms for indifference graphs, Comput. Math. Appl., 25 (1993), 15-25.

[19] K. Matsuda, Weakly closed graphs and F-purity of binomial edge ideals, (2012) arXiv:1209.4300v1.

[20] D. Meister, Recognition and computation of minimal triangulations for AT-free claw-free and co-comparability graphs, Discrete Appl. Math., 146 (2005), 193-218.

[21] M. Ohtani, Graphs and ideals generated by some 2-minors, Comm. Algebra, 39 (3) (2011), 905-917.

[22] B. S. Panda, S. K. Das A linear time recognition algorithm for proper interval graphs, Inform. Process. Lett., 87 (2003), 153-161. 
[23] F.S. Roberts, Indifference graphs, In Proof Techniques in Graph Theory (F. Harary, ed.), (1969), 139-146. Academic Press, New York, NY.

[24] D. Rose, G. Lueker, R. E. Tarjan, Algorithmic aspects of vertex elimination on graphs, SIAM J. Comput., 5 (1976), 266-283.

[25] M.S. Waterman, J.R. Griccs, Interval graphs and maps of DNA, Bull. Math. Biol., 48 (1986), 189-195.

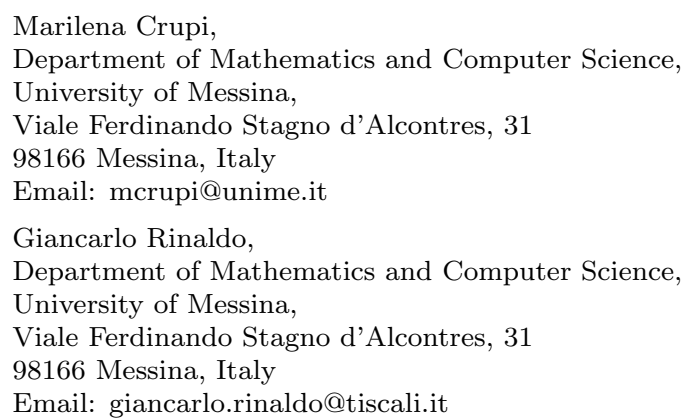

Email: giancarlo.rinaldo@tiscali.it 